\title{
L'HOMME L'Homme
}

Revue française d'anthropologie

$183 \mid 2007$

Comment être parents ?

\section{Alessandro Duranti, ed., A Companion to Linguistic Anthropology \\ Malden-Oxford, Blackwell, 2006, 625 p., bibl., index, ill., fig. (« Blackwell Companion to Anthropology »)}

\section{Pierre Déléage}

\section{(2) OpenEdition}

\section{Journals}

Édition électronique

URL : http://journals.openedition.org//homme/9711

DOI : 10.4000/lhomme.9711

ISSN : 1953-8103

Éditeur

Éditions de l'EHESS

\section{Édition imprimée}

Date de publication : 1 septembre 2007

Pagination : 211-213

ISSN : 0439-4216

Référence électronique

Pierre Déléage, «Alessandro Duranti, ed., A Companion to Linguistic Anthropology », L'Homme [En ligne], 183 | 2007, mis en ligne le 28 juin 2007, consulté le 24 septembre 2020. URL : http:// journals.openedition.org//homme/9711; DOI : https://doi.org/10.4000//homme.9711

Ce document a été généré automatiquement le 24 septembre 2020

(c) École des hautes études en sciences sociales 


\section{Alessandro Duranti, ed., A Companion to Linguistic Anthropology}

Malden-Oxford, Blackwell, 2006, 625 p., bibl., index, ill., fig. (« Blackwell Companion to Anthropology »)

\section{Pierre Déléage}

1 CONTRAIREMENT À LA FRANCE, où les anthropologues restent, aujourd'hui encore, méfiants vis-à-vis des travaux des linguistes, qu'ils jugent quelque peu ésotériques, les États-Unis n'ont cessé de cultiver un champ interdisciplinaire qu'ils nomment l'anthropologie linguistique. Le champ n'est certes pas dominant dans un univers académique gigantesque où la principale manifestation, le congrès de l'American Anthropological Association, regroupe des milliers de participants intervenant dans des centaines de symposiums aux intitulés les plus variés et parfois les plus inattendus. Il n'en reste pas moins à la fois important et prestigieux, et réunit des anthropologues capables de publier à la fois des articles notables dans des revues de linguistiques (comme, par exemple, l'International Journal of American Linguistics) et des contributions souvent originales dans les principaux périodiques d'anthropologie générale. Par ailleurs, tandis qu'une bonne partie du monde académique américain considère les anthropologues européens, qui s'intéressent encore à des phénomènes qu'ils osent qualifier de «traditionnels» (avec ou sans guillemets), comme les derniers tenants d'une pratique archaïque sinon condamnable, les anthropologues linguistes persistent à accorder à la description ethnographique et en particulier à la transcription attentive de discours (quels qu'ils soient) une certaine légitimité. La lecture de leurs textes apporte donc, souvent, plus d'informations sur les sociétés dont ils traitent que sur les spéculations propres à leur auteur, ce qui n'est plus si commun.

2 L'anthropologie linguistique possède ainsi une véritable existence institutionnelle aux États-Unis qui se traduit aussi bien par la société au sein de laquelle ils se reconnaissent, la Society for Linguistic Anthropology, que par les lointaines racines historiques de la discipline. Les anthropologues linguistes s'inscrivent en effet, nolens volens, dans une longue tradition proprement américaine dont plusieurs jalons remontent au xIX siècle (de Thomas Jefferson au Bureau of American Ethnology du Major 
John Wesley Powell). C'est cependant Franz Boas qui institutionnalisa, dans son pays d'adoption, l'approche linguistique en anthropologie. Celui-ci, qui a joué le rôle que l'on sait dans la professionnalisation de l'anthropologie américaine à travers son influence sur la mise en place de cursus spécialisés au sein du système universitaire, voyait sa discipline caractérisée par quatre champs, certes distincts, mais qui devaient selon lui rester complémentaires: l'anthropologie culturelle, l'anthropologie physique, l'archéologie et la linguistique. La discipline a beaucoup changé depuis les directives de Boas, qui a néanmoins initié un courant ininterrompu, où les changements de paradigmes hautement proclamés cachent une forte continuité institutionnelle, qui va d'Edward Sapir à Michael Silverstein en passant par Dell Hymes. En digne héritier de cette tradition, Alessandro Duranti, professeur à l'UCLA, a beaucoup œuvré au cours des dernières années pour rendre l'anthropologie linguistique à la fois plus visible et plus conquérante sur « le marché américain ».

Le Companion to Linguistic Anthropology répond à ces objectifs, et il vient à la suite d'un textbook ${ }^{1}$, d'un dictionnaire des principaux concepts de la discipline ${ }^{2}$ et d'une sélection d'articles du champ devenus, comme on dit, «incontournables " ${ }^{3}$. Ce nouveau recueil, destiné certes aux étudiants mais qui intéressera, ponctuellement ou dans son intégralité, tous ceux qui s'interrogent parfois sur l'inévitable dimension linguistique de leur travail, regroupe des contributions d'auteurs aussi bien confirmés que relativement débutants.

D'une manière générale, l'anthropologie linguistique se distingue de l'anthropologie par une attention décuplée accordée aux phénomènes langagiers et de la linguistique par une prise en compte du contexte des usages de la parole (ses figures tutélaires étrangères sont Benveniste ou Bakhtine). Les concepts élaborés sont ainsi toujours pensés en fonction de cette double exigence de démarcation et ils permettent parfois de construire des objets d'étude dont la richesse commence tout juste à être soupçonnée. Ils éclairent sous un nouvel angle des problèmes classiques comme les interactions inégalitaires, les contacts, échanges ou conflits entre cultures différentes, les pratiques instituées telles que les narrations traditionnelles et les rituels, ou les principes de la cognition la plus ordinaire telle qu'elle se manifeste dans les conversations quotidiennes. Ce Companion permettra au lecteur curieux de faire le point sur chacun de ces éclairages, afin, peut-être, de les mettre à profit dans son propre travail.

5 Les vingt-deux chapitres - tous, sauf un, rédigés par des Américains - sont consacrés soit à des thématiques générales envisagées selon le point de vue propre à l'anthropologie linguistique (Îles du Pacifique, Langues d'Amérique du Nord, Langage des signes, Conversation, Gestualité, Literacy, Poétique, Anthropologie de la voix, Identité, Malentendus, Folie, Religion, Inégalité sociale), soit à des concepts qui sont comme la signature du champ (Speech community, Registre de langue, Contact interlinguistique, Codeswitching, Participation, Schémas narratifs, Socialisation linguistique, Agency, Idéologie linguistique). La bibliographie, très riche, fournira à celle ou celui qui ne se satisfait pas de ces aperçus, les indications nécessaires pour explorer ce domaine en constante expansion.

6 La lecture de ce Companion permet, par ailleurs, de vérifier que l'anthropologie linguistique se méfie toujours des théories ambitieuses du langage et de la communication $^{4}$. Son apport se situe volontairement en marge des recherches en pragmatique (de Steven Levinson ou de Dan Sperber et Deirdre Wilson), en linguistique 
«fonctionnelle » (par exemple, Michael Tomasello) ou en anthropologie cognitive. Elle préfère définir précisément, à l'aide d'exemples très détaillés, des séries d'outils conceptuels susceptibles d'offrir des éclairages partiels mais pertinents de tel ou tel aspect de l'activité humaine en général. Elle s'est ainsi depuis longtemps alliée aux tenants de disciplines connexes, qui partagent souvent les mêmes références et la même inspiration; on pense en particulier au continuum qui va de la sociologie de l'interaction à l'analyse conversationnelle en passant par l'ethnométhodologie (représentée par Erving Goffman, John J. Gumperz, Aaron V. Cicourel ou Emanuel A. Schegloff). Il en résulte une grande prudence méthodologique : chacun des concepts reconnaissables de l'anthropologie linguistique a fait couler des quantités d'encre impressionnantes et les discussions peuvent paraître, vues de France, un tantinet scolastiques. Mais cette hypocondrie épistémologique n'est pas stérile : la plupart des grands ethnographes américains de ces dernières années ont été, à un moment ou à un autre de leur carrière, des anthropologues linguistes. Ce livre constitue donc une porte d'entrée idéale pour tous ceux qui veulent découvrir les idées actuelles et les propositions de recherche de l'anthropologie linguistique. Il devrait surtout être utile aux étudiants et sa lecture ne manquera pas de les rendre attentifs à des aspects trop souvent laissés de côté par les anthropologues français.

\section{NOTES}

1. Alessandro Duranti, ed., Linguistic Anthropology, New York, Cambridge University Press, 1997.

2. Alessandro Duranti, ed., Key Terms in Language and Culture, Malden, Blackwell Publishing, 1999-2001.

3. Alessandro Duranti, ed., Linguistic Anthropology : A Reader, Malden, Blackwell, 2001.

4. Pour une critique similaire, à partir d'une perspective différente, voir Michael Silverstein, « How We Look from Where We Stand », Journal of Linguistic Anthropology, 2006, 16 (2) : 269-278.

\section{AUTEUR \\ PIERRE DÉLÉAGE \\ University of California, Department of Anthropology, Berkeley. \\ deleagepierre@hotmail.com}

